\title{
Towards an understanding of third-order galaxy-galaxy lensing (Corrigendum)
}

\author{
Patrick Simon, Peter Schneider, and Daniela Kübler
}

\author{
Argelander-Institut für Astronomie, Universität Bonn, Auf dem Hügel 71, 53121 Bonn, Germany \\ e-mail: psimon@astro.uni-bonn.de
}

A\&A 548, A102 (2012), DOI: 10.1051/0004-6361/201218993

Key words. gravitational lensing: weak - galaxies: halos - large-scale structure of Universe - errata, addenda

We report several confusing typographical errors in our original publication (Simon et al. 2012). In four instances, the term lens-shear-shear was accidentally replaced by lens-lens-shear, which is also present in the same publication but with a different meaning.

A detailed list of the typographical errors follows:

1. In the abstract on page 1 , the sentence

"The lens-lens-shear correlator is sensitive to variations among matter haloes." should be:

"The lens-shear-shear correlator is sensitive to variations among matter haloes."

2. At the end of the introduction on page 2, the sentence

"Section 4 does the same for the lens-lens-shear or $G_{ \pm}$correlator." should be:

"Section 4 does the same for the lens-shear-shear or $G_{ \pm}$correlator."

3. The heading of Sect. 4 on page 8 ,

"4. Lens-lens-shear correlator", should be:

"4. Lens-shear-shear correlator"

4. In Appendix A on page 12, before Eq. (A.6), the sentence "Similarly, we find for the lens-lens-shear correlator..."

should be:

"Similarly, we find for the lens-shear-shear correlator..."

Acknowledgements. We thank our colleague Stefan Hilbert for pointing out the typos in our original publication. 